Anje Ostermann, Anke Lindmeier, Hendrik Härtig, Lorenz Kampschulte, Mathias Ropohl \& Julia Schwanewedel

\title{
Mathematikspezifische Medien nutzen: Was macht den Unterschied - Lehrkraft, Schulkultur oder Technik?
}

\section{Zusammenfassung}

Forschung zu Mediennutzung im Unterricht nimmt bisher selten einen fachlichen Blick ein, obwohl gerade im Mathematikunterricht spezifische Medien im Einsatz sind. Die berichtete Lehrkräftebefragung zeigt die aktuelle mathematikspezifische Mediennutzung in der Sekundarstufe auf und erklärt Unterschiede durch individuelle und organisationale Faktoren im Kontrast unterschiedlicher Gruppen von Nutzungshäufigkeit. Weiter diskutieren wir Implikationen zur Förderung von Medieneinsatz im Mathematikunterricht. Schlüsselwörter: Mathematikunterricht, Medieneinsatz, Lehrkräfte, Schulkultur

\section{Using Mathematics-specific Media: What Makes the Difference - Teachers, School-Culture, or Equipment?}

\begin{abstract}
Research on media use in teaching has as yet rarely taken a subject-specific view, despite the fact that specific tools are used in mathematics instruction in particular. The reported teacher survey shows the current mathematics-specific media use in secondary level education in Germany and explains differences by individual and organizational factors within different groups of usage frequency. Furthermore, we discuss implications for promoting media use in mathematics instruction.

Keywords: mathematics education, media use, teachers, school-culture
\end{abstract}

\section{$1 \quad$ Einleitung}

In der Schule wächst die Rolle digitaler Medien (KMK, 2016), wobei die Digitalisierung als schulische Querschnittsaufgabe gesehen wird (van Ackeren et al., 2019). Untersuchungen zur Mediennutzung folgen entsprechend meist einem fachübergrei- 
fenden Blick, etwa werden Merkmale wie die Ausstattung an der Schule oder deren Nutzung ohne Fachunterscheidung beschrieben (z.B. Eickelmann et al., 2019; BITKOM, 2015). Gleichwohl ist Schule überwiegend in Unterrichtsfächern strukturiert, was zur Folge hat, dass auch eine Querschnittsaufgabe letztlich im Fachunterricht realisiert werden muss. Nur wenige Studien nehmen bisher jedoch eine spezifischere Perspektive ein und untersuchen, wie Medien im (MINT-)Fachunterricht integriert werden (z.B. Hillmayr, Reinhold, Ziernwald \& Reiss, 2017; Lorenz, Bos \& Endberg, 2017).

Dies ist umso verwunderlicher, als aus fachlicher Sicht der sinnvolle Einsatz spezifischer Werkzeuge als wichtig erachtet wird (Härtig, Kampschulte, Lindmeier, Ostermann, Ropohl \& Schwanewedel, 2018). Im Fach Mathematik sollen z. B. dynamische Geometrie-Systeme (DGS, ermöglichen die Dynamisierung von Konstruktionen), Tabellenkalkulationsprogramme (TKP, ermöglichen die Verarbeitung numerischer Daten in Tabellenform) oder Computer-Algebra-Systeme (CAS, ermöglichen die exakte Umformung algebraischer Ausdrücke), eingesetzt und der Umgang mit ihnen geübt werden (KMK, 2015). Die Nutzung dieser Medien ist vermutlich, ebenso wie die Mediennutzung im Allgemeinen, von Merkmalen auf Ebene der Lehrkräfte (z. B. Selbstwirksamkeitserwartung, Einstellungen) und Ebene der Schule (z. B. Ausstattung, schulinterne Regelungen) abhängig (Prasse, 2012). Es ist aber anzunehmen, dass Merkmale auf Ebene der Schul-Fachkultur ebenso eine Rolle spielen (Barzel, 2012). Bisher gibt es dazu jedoch kaum Untersuchungen und so bleibt es ungewiss, ob die fachunabhängig gewonnenen Erkenntnisse zur Mediennutzung, sowie deren Bedingungsfaktoren auf organisationaler Ebene und Ebene der Lehrkräfte auch für fachspezifische Medien gelten.

Daher ist ein Ziel der hier vorgestellten Studie, unter einem stärker fachspezifischen Blick zu erfassen, wie sich der Einsatz mathematikspezifischer Medien im Unterricht am Beispiel von CAS und DGS darstellt. Darüber hinaus sollen Einflüsse der organisationalen Rahmenbedingungen und der Merkmale von Lehrpersonen auf den fachspezifischen Medieneinsatz untersucht werden, um geeignete Ansatzpunkte für die Lehrkräfteaus- und -weiterbildung im Bereich des Medieneinsatzes im Mathematikunterricht $\mathrm{zu}$ identifizieren.

\section{Theoretischer Hintergrund}

Mathematikunterricht mit digitalen Medien ist Gegenstand vieler Untersuchungen: Häufig steht dabei die Frage danach, wie Schüler*innen mathematische Inhalte mithilfe digitaler Medien lernen, im Fokus (u.a. Hoffkamp, 2011; Rieß, 2018; Weigand \& Bichler, 2010). So untersuchen Rolfes und Kollegen (2020) bspw. die Wirkung verschiedener Visualisierungen mathematischer Inhalte (statisch, dynamisch und dyna- 
misch mit Interaktionsmöglichkeit) auf das Lernen im Bereich Funktionen und finden dabei einen Vorteil dynamischer Visualisierungen.

Andere Arbeiten bearbeiten die Frage nach der Gestaltung digitaler Medien im Mathematikunterricht und damit einhergehend der Strukturierung und den Realisierungen von Lerninhalten mithilfe digitaler Medien. So finden sich einige Untersuchungen, die das Design mediengestützter Aufgaben oder umfassenderer mathematischer Lernumgebungen in den Blick nehmen (u. a. Fahlgren \& Brunström, 2014; Venturini \& Sinclair, 2017).

Erkenntnisse solcher Untersuchungen tragen dazu bei, eine wirkungsvolle Gestaltung und Integration digitaler Medien im Mathematikunterricht $\mathrm{zu}$ verstehen und zu fördern. Ob digitale Medien in den Mathematikunterricht integriert werden und welche Faktoren dafür relevant sind, lässt sich daraus jedoch nur bedingt ableiten. Allerdings können verschiedene allgemeine Ansätze zur Beschreibung des Technologieeinsatzes in der Schule Hinweise auf Antworten zu diesen Fragen liefern. Prasse (2012) arbeitet heraus, dass diese Ansätze - wenn auch in unterschiedlicher Feinkörnigkeit - ähnliche Einflussfaktoren annehmen, die auf mehreren Ebenen wirken. Dabei lässt sich ein Teil der Einflussfaktoren für die Nutzung von Technologien auf der individuellen Ebene der Lehrkraft verorten. Andere können als organisationale Merkmale der Schule charakterisiert werden.

Auf der individuellen Ebene findet sich allgemein ein positiver Zusammenhang zwischen der Nutzungshäufigkeit von Informations- und Kommunikations-Technologien (IKT), wie z.B. Computer, im Unterricht und den Einstellungen von Lehrkräften (u. a. Hermans, Tondeur, van Braak \& Valcke, 2008; Petko, 2012; Prasse, 2012). Auch die Selbstwirksamkeitserwartung der Lehrkräfte in Bezug auf das Unterrichten mit IKT scheint einen positiven Effekt auf die Nutzungshäufigkeiten von IKT im Unterricht zu haben (u.a. Drossel, Eickelmann \& Gerick, 2017; Petko, 2012). Dieser Zusammenhang konnte auch für den Mathematikunterricht international (u.a. Thomas \& Palmer, 2014; Handal, Cavanagh, Wood \& Petocz, 2011) und im deutschen Kontext (Thurm, 2020) gezeigt werden.

Auf der organisationalen Ebene der Schule gibt es Hinweise, dass die quantitative Ausstattung mit IKT einen positiven Effekt auf die Nutzungshäufigkeit im Unterricht hat (Petko, 2012). Eickelmann, Gerick und Koop (2017) finden diesen Zusammenhang auch speziell für den Mathematikunterricht. Petko (2012) führt jedoch an, dass die reine Anzahl verfügbarer Computer nur bedingt aussagekräftig zur Beurteilung der Ausstattung ist. Ob die Geräte problemlos zugänglich im Klassenraum sind oder nur in Computerräumen zur Verfügung stehen, kann z.B. einen Unterschied machen. Prasse (2012), die in die Beurteilung der Ausstattung neben der Quantität auch qualitative Aspekte der Organisation (z. B. unkomplizierte Zugangsregeln, keine zusätzlichen Absprachen notwendig) einbindet, findet jedoch 
keinen Zusammenhang der Ausstattung mit der Nutzungshäufigkeit im Unterricht. Ein einheitliches Bild zeigt sich an dieser Stelle also nicht. Zuletzt zeigen schulinterne Regelungen wie z.B. eine IKT-Strategie positive Effekte auf die Nutzung von IKT im Unterricht (Prasse, 2012; Tondeur, van Keer, van Braak \& Valcke, 2008).

Eine Untersuchung, die die Bedeutung individueller und organisationaler Merkmale für IKT-Nutzung gleichzeitig berücksichtigte, wurde zu Beginn der 2000er Jahre durchgeführt (Prasse, 2012). Sie konnte zeigen, dass neben den persönlichen Lehrkraftmerkmalen (wie IKT-bezogene Einstellungen, wahrgenommene Kompetenzen) auch organisationale Merkmale (wie gemeinsame Ziele und Visionen an einer Schule, Unterstützung durch die Schulleitung) einen positiven Effekt auf die Nutzung von IKT im Unterricht haben. Individuelle und organisationale Faktoren hängen also in einem komplexen Wechselspiel mit der Nutzungshäufigkeit zusammen.

\section{Forschungsfragen}

Bisherige Studien legen nahe, dass individuelle Lehrkraftmerkmale (wie Kompetenz, Einstellungen und Werthaltungen in Bezug auf Medien) und organisationale Merkmale (wie Zugang oder Schulklima) einen Effekt auf den Einsatz von Medien im Unterricht haben. Empirische Befunde dazu, ob sich dies für die Nutzung fachspezifischer Medien ebenso darstellt wie für die Nutzung von IKT aus genereller Sicht, sind bisher jedoch rar. Besonders für das Fach Mathematik gibt es keine verlässlichen Daten zur tatsächlichen Nutzung oder Analysen zu deren Bedingungsfaktoren. Deswegen können kaum spezifische evidenzbasierte Ansatzpunkte für die Unterstützung der Integration mathematikspezifischer digitaler Medien in den Unterricht abgeleitet werden.

Vor diesem Hintergrund wird in dieser Studie zunächst mithilfe einer Lehrkräftebefragung erhoben, wie sich das aktuelle Nutzungsverhalten am Beispiel von CAS und DGS als mathematikspezifische Medien mit unterschiedlichem Verpflichtungsgrad (DGS: verpflichtend, CAS: teils verpflichtend) im Mathematikunterricht der Sekundarstufe I darstellt (Forschungsfrage 1). Aufbauend darauf soll untersucht werden, inwiefern Personenmerkmale und/oder organisationale Merkmale Unterschiede im Nutzungsverhalten in Bezug auf CAS und DGS erklären können (Forschungsfrage 2). 


\section{Methode}

\subsection{Stichprobe}

Insgesamt wurden $N=163$ Mathematiklehrkräfte, die in der Sekundarstufe I unterrichten, zum Medieneinsatz im Mathematikunterricht befragt (Zeitraum April 2017 - September 2018, wahlweise papierbasiert oder online). Die Lehrkräfte wurden auf Lehrkräftetagungen und über Schulen direkt kontaktiert. Der Großteil der Befragten stammt aus Nordrhein-Westfalen (47\%) und Schleswig-Holstein (33\%). In diesen Bundesländern sind DGS im Unterricht der Sekundarstufe I verpflichtend, die Nutzung von CAS ist optional (Schleswig-Holstein) bzw. nicht in den Lehrplänen aufgeführt (Nordrhein-Westfalen). Daher ist davon auszugehen, dass CAS deutlich seltener genutzt werden als DGS. Die Mehrheit der Lehrkräfte unterrichtet an einem Gymnasium (61 \%). In Anlehnung an Phasenmodelle der Entwicklung professioneller Kompetenz (z.B. Huberman, 1999) wurde die Berufserfahrung zusammengefasst in fünf Phasen erhoben (vgl. Abb. 1).

Abb. 1: Berufserfahrung der Stichprobe $(N=163)$

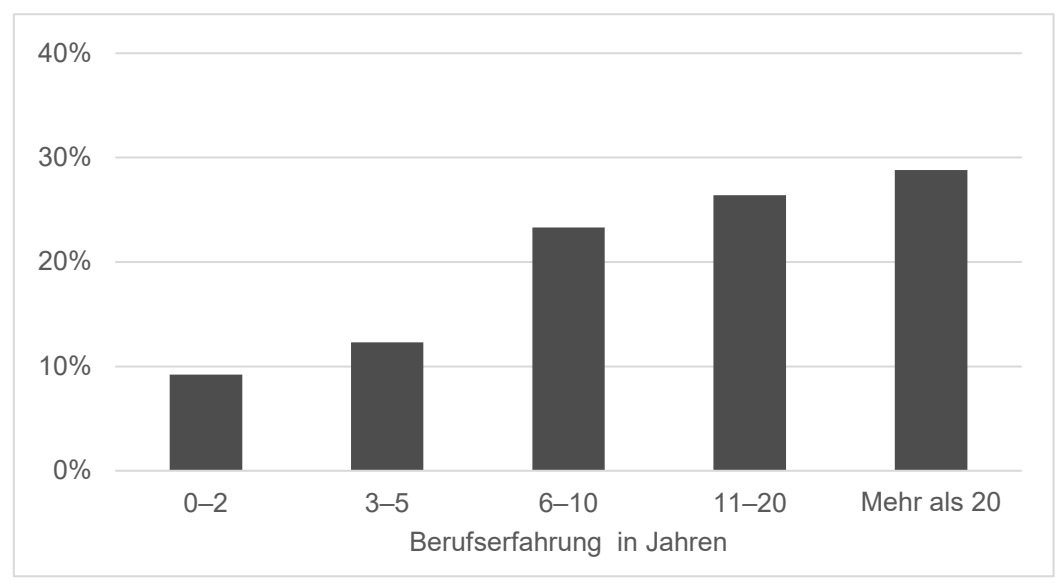

Quelle: eigene Darstellung

Die Gelegenheitsstichprobe wurde mit der Stichprobe einer repräsentativen Untersuchung von Lehrkräften aller Fächer mithilfe zweier Indikatoren aus der BITKOMStudie von 2014 (BITKOM, 2015) verglichen, um Verzerrungen abzuschätzen: In Bezug auf das Verhältnis zu digitalen Medien generell (4-stufiges Antwortformat: 1 = „Ich kann mit digitalen Medien nichts anfangen“, 4 = „Ich bin ein Technik-Fan“) zeigen sich die Mathematiklehrkräfte aus der vorliegenden Studie im Schnitt positiver als die BITKOM-Lehrkräfte $(\Delta=0.13, \mathrm{t}(280.27)=2.69, p=0.008, d=0.32)$. Dem Einsatz digitaler Medien im Unterricht gegenüber (4-stufig: $1=$ „negativ“, $4=$,positiv") zeigen sich die Mathematiklehrkräfte jedoch skeptischer $(\Delta=-0.36$, 
$\mathrm{t}(660)=-6.48, p<0.001, d=-0.50)$. Die Unterschiede sind als kleiner bzw. mittlerer Effekt zu charakterisieren und statistisch signifikant. Die gegensätzlichen Richtungen der Abweichungen lassen keinen Schluss auf Repräsentativität unserer Stichprobe zu, weisen aber auch nicht auf eine systematische positive Verzerrung hin.

\subsection{Instrumente}

In der Befragung wurden individuelle Merkmale auf Ebene der Lehrkräfte, organisationale Merkmale auf Ebene der Schulen sowie die Nutzung der mathematikspezifischen Medien durch die Lehrkräfte erhoben. Dazu konnten teils bestehende Instrumente genutzt oder angepasst werden (vgl. Tab. 1).

Tab. 1: Übersicht erhobener Merkmale und eingesetzter Instrumente

\begin{tabular}{lll}
\hline & Merkmal & Instrument \\
\hline $\begin{array}{l}\text { Individualebene } \\
\text { Lehrkräfte }\end{array}$ & $\begin{array}{l}\text { Einstellungen } \\
\text { zum Einsatz digitaler Medien im Unterricht }\end{array}$ & $\begin{array}{l}\text { Lindau, Kübler \& } \\
\text { Spada, 2013 }\end{array}$ \\
\cline { 2 - 3 } & $\begin{array}{l}\text { Selbstwirksamkeitserwartung } \\
\text { in Bezug auf die Planung und Durchführung von } \\
\text { Unterricht mit digitalen Medien }\end{array}$ & $\begin{array}{l}\text { Adaption nach } \\
\text { Meinhardt, Rabe } \\
\text { \& Krey, 2016 }\end{array}$ \\
\hline $\begin{array}{l}\text { Organisationale } \\
\text { Ebene }\end{array}$ & $\begin{array}{l}\text { Zugang } \\
\text { zu CAS/DGS }\end{array}$ & Eigenentwicklung \\
\cline { 2 - 3 } & $\begin{array}{l}\text { Schulinterne Rege/ungen } \\
\text { bzgl. genutzter CAS-/DGS-Geräte }\end{array}$ & Eigenentwicklung \\
\hline Nutzung & $\begin{array}{l}\text { Nutzungshäufigkeit } \\
\text { bestimmter CAS-/DGS-Lernaktivitäten durch die }\end{array}$ & Eigenentwicklung \\
& $\begin{array}{l}\text { Schüler*innen } \\
\text { (bei typischen Themen) }\end{array}$ & \\
\hline
\end{tabular}

Quelle: eigene Darstellung

Die Einstellungen zum Einsatz digitaler Medien im Unterricht wurden mithilfe einer sechs Items umfassenden Skala erhoben (z. B. „Digitale Medien sorgen im Unterricht für Unruhe und lenken vom Lerninhalt ab“, 5-stufiges Antwortformat: $1=$ „stimme überhaupt nicht zu“, $5=$ „stimme voll zu“, Lindau, Kübler \& Spada, 2013). Die Selbstwirksamkeitserwartungen in Bezug auf die Planung und Durchführung von Unterricht mit digitalen Medien wurde mit einer aus sechs Items bestehenden Skala erfasst. Die Skala der Selbstwirksamkeitserwartung wurde adaptiert nach der physikspezifischen Lehrkräfteselbstwirksamkeitserwartung nach Meinhardt, Rabe und Krey (2016), wobei der Begriff der Selbstwirksamkeitserwartung angelehnt an Schmitz und Schwarzer (2000) als eine Handlung, die mit einer Schwierigkeit verbunden ist, spezifiziert wurde (z.B. „Ich kann den fachlichen Lernprozess durch den Einsatz digitaler Medien unterstützen, auch wenn unvorhergesehene Verständnisschwierigkeiten auftreten“; 4-stufiges Antwortformat: $1=$ „stimmt nicht“, $4=$ „stimmt genau“). Die 
Skalen Einstellungen und Selbstwirksamkeitserwartung beziehen sich auf digitale Medien allgemein und wurden entsprechend nicht spezifisch für CAS und DGS erfasst. Die Skala „Selbstwirksamkeitserwartung beim Unterrichten mit digitalen Medien" befindet sich im Anhang.

Der Zugang zu CAS und DGS als ein Merkmal schulischer Rahmenbedingungen wurde mithilfe je einer Frage erhoben. Es musste aus fünf typischen Optionen diejenige gewählt werden, die die Situation an der eigenen Schule am besten spiegelt (z. B. „DGS sind für alle Lernenden bei Bedarf als Klassensatz verfügbar", vgl. Abb. 2 auf S. 207). Von Lehrkräften ohne Zugang wurden keine weiteren Angaben zu diesem Medium, insbesondere auch keine Nutzungshäufigkeiten, erhoben. Damit wurde in dieser Studie für das Merkmal Zugang nicht (allein) die quantitative Ausstattung an Geräten gewählt.

Weiter wurde ein einfacher Indikator zu schulinternen Regelungen erhoben („An meiner Schule gibt es eine vs. gibt es keine einheitliche CAS/DGS-Regelung"). Solche Regelungen sollen in vielen Bundesländern von den Fachkonferenzen festgelegt werden (z. B. in Schleswig-Holstein) und etwa die Rechtsicherheit bei Prüfungen gewährleisten. Es kann davon ausgegangen werden, dass der Medieneinsatz an Schulen mit einheitlicher Regelung grundlegender und verbindlicher geklärt ist als an Schulen ohne erarbeitete Regelungen. Daher wurde das Vorhandensein schulinterner Regelungen bzgl. der genutzten Geräte für CAS und DGS als Indikator für fachspezifische schulinterne Rahmenbedingungen erhoben, der über den reinen Zugang hinaus informativ für das fachspezifische Medien-Schulklima ist.

Die Mediennutzungshäufigkeit in Bezug auf CAS und DGS wurde in Eigenentwicklung operationalisiert. Ähnlich wie bei Sailer, Mürbock und Fischer (2017) wurden Lernaktivitäten von Schülerinnen und Schülern mit DGS/CAS identifiziert, die die verschiedenen Funktionen schulischer Mediennutzung abbilden (Beitrag zur Grundbildung, Entwicklung von Medienkompetenz, instrumentelle Nutzung im Fachunterricht; Kerres, 2000). Die Lernaktivitäten im Bereich der instrumentellen Nutzung wurden dabei im Anschluss an den mathematikdidaktischen Diskurs bestimmt: Während das Erstellen eigener Berechnungen/Konstruktionen und deren Überprüfung grundlegende Funktionen nutzt, besteht das Potenzial von CAS und DGS darin, Visualisierungen zu erstellen sowie Gesetzmäßigkeiten zu entdecken (zsf. Schmidt-Thieme \& Weigand, 2010). Die Erhebung der Nutzung als Häufigkeit dieser Lernaktivitäten wurde je an ein kanonisches, für den Medieneinsatz besonders geeignetes Themenbeispiel (DGS: Strahlensätze, CAS: Lösen quadratischer Gleichungen) gekoppelt, um eine möglichst gute Vergleichbarkeit zwischen den Angaben der Lehrkräfte $\mathrm{zu}$ erzielen. Für jede der Lernaktivitäten wurden die Lehrkräfte aufgefordert, die auf die Unterrichtseinheit bezogenen Nutzungshäufigkeiten anzugeben (vgl. Tab. 2 auf der folgenden Seite). Dabei wurde eine Skalierung gewählt, die die typische Planungseinheit „Unterrichtsstunde“ nutzt ( $1=$ „in keiner oder fast keiner“", 2 = „in 
weniger als der Hälfte“, 3 = „in mindestens der Hälfte“, 4 = „in jeder oder fast jeder Unterrichtsstunde“). Weitere Lernaktivitäten konnten über offene Antwortformate ergänzt werden.

Tab. 2: Übersicht über die Konzeption der Lernaktivitäten zur Erhebung der Nutzungshäufigkeit

\begin{tabular}{ll}
\hline Fokus & Lernaktivität \\
& (Frage: „Wie häufig führen Ihre Schülerinnen und Schüler folgende \\
& $\begin{array}{l}\text { Lernaktivitäten typischerweise bei der Nutzung von DGS / CAS im } \\
\text { Mathematikunterricht zum Thema "'Strahlensätze” / „Lösen } \\
\text { quadratischer Gleichungen” durch?") }\end{array}$ \\
\hline Instrumentelle & zum Überprüfen eigener Berechnungen. \\
Nutzung & zur Konstruktion / Berechnung einer Lösung. \\
& zur Visualisierung eines Sachverhalts. \\
& zum Herausfinden von Zusammenhängen und Regeln. \\
Grundbildung & zum Lernen der Bedienung des DGS/CAS. \\
Medienkompetenz & zur Reflexion des mathematischen Hintergrunds der DGS-/CAS- \\
& Funktionalitäten. \\
\hline
\end{tabular}

Quelle: eigene Darstellung

\subsection{Methode}

Um die Frage nach der Nutzungshäufigkeit zu beantworten, wurden die Angaben zu CAS bzw. DGS aggregiert (vgl. Kap. 5.2). In einem zweiten Schritt wurde eine multinomiale logistische Regression (MLR) durchgeführt (abhängige Variable: Nutzungshäufigkeit), um die Effekte von Personenmerkmalen und organisationalen Merkmalen zu untersuchen (Variableneinschluss schrittweise vorwärts).

\section{Ergebnisse}

\subsection{Deskriptiva}

Für CAS gaben 41 Lehrkräfte an, über keinen Zugang zu verfügen, bei DGS waren es drei Lehrkräfte. Abbildung 2 auf der folgenden Seite zeigt die Häufigkeitsverteilungen der Angaben zum CAS- bzw. DGS-Zugang für die Lehrkräfte mit Zugang. Die Antworten wurden für die weitere Verwendung gruppiert. Dabei wurde unterschieden, ob für die Lehrkräfte der Einsatz von CAS bzw. DGS mit einem erhöhten unterrichts-organisatorischen Aufwand verbunden ist (Code 0, Buchung/Umzug in den Computerraum notwendig oder eingeschränkte Geräteverfügbarkeit) oder ob es keine erhöhte Zugangshürde gibt (Code 1). 
Abb. 2: Häufigkeitsverteilung der Angaben zum Zugang zu CAS $(N=144)$ bzw. DGS $(N=160)$

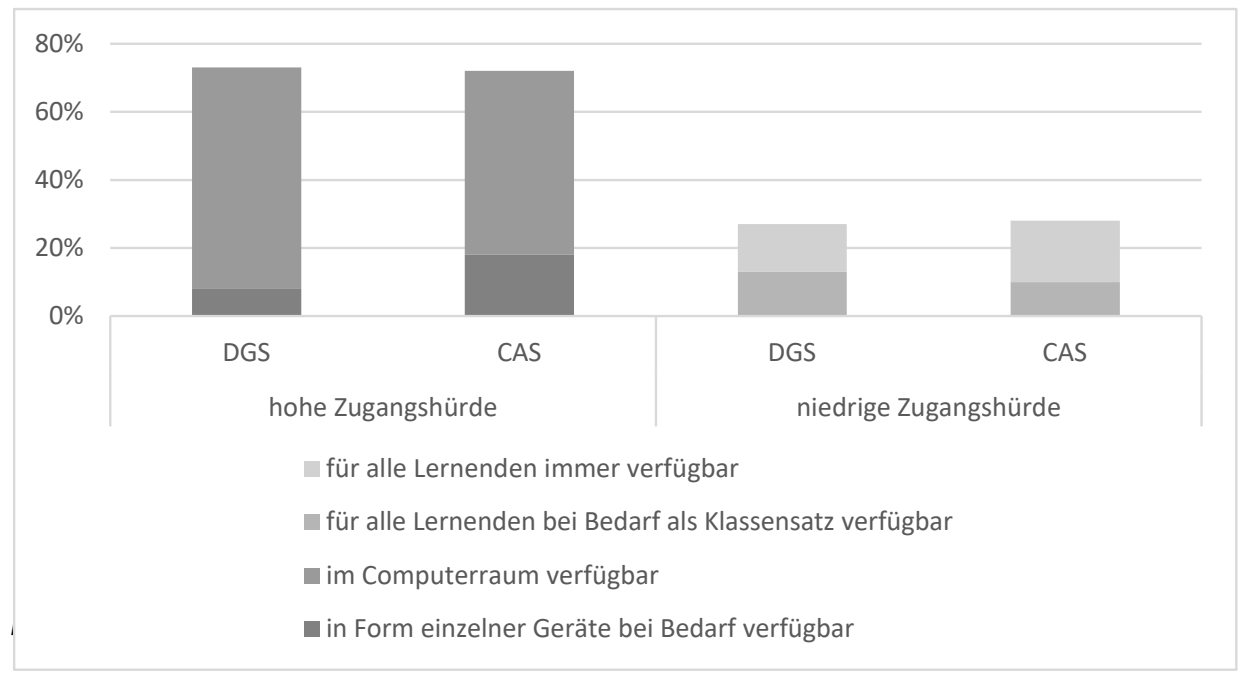

Quelle: eigene Darstellung

Dass an ihren Schulen keine schulinternen Regelungen bezüglich der Gerätewahl bestehen, berichten 40,4 Prozent (für CAS) bzw. 43,4 Prozent (für DGS) der Lehrkräfte.

Die Mittelwerte und Standardabweichungen der Skalen zu Einstellungen (5-stufiges Antwortformat) und Selbstwirksamkeitserwartung (4-stufig) gegenüber dem Einsatz digitaler Medien im Unterricht sind in Tabelle 3 aufgeführt. Die Angaben zu den Einstellungen sind hoch, sodass sich ein leichter Deckeneffekt zeigt.

Tab. 3: Mittelwerte und Standardabweichungen der Skalen Einstellungen und Selbstwirksamkeitserwartung

\begin{tabular}{lcccccc}
\hline Skala & $\begin{array}{c}\text { Item- } \\
\text { anzahl }\end{array}$ & $\begin{array}{c}\text { Cronbachs } \\
\text { Alpha }\end{array}$ & MW & SD & Minimum & Maximum \\
\hline Einstellungen & 6 & .83 & 3.96 & 0.66 & 2 & 5 \\
$\begin{array}{l}\text { Selbstwirksam- } \\
\text { keitserwartungen }\end{array}$ & 6 & .77 & 2.77 & 0.54 & 1 & 4 \\
\hline
\end{tabular}

Quelle: eigene Berechnung 


\subsection{Wie stellt sich die Nutzungshäufigkeit von CAS und DGS dar (Forschungsfrage 1)?}

In die Auswertung gingen nur Daten von Lehrkräften ein, die Zugang zu DGS bzw. CAS haben $\left(n_{C A S}=103, n_{D G S}=156\right)$. Die Kategorie 4 (,in jeder oder fast jeder Unterrichtsstunde“) war kaum besetzt, sodass sie mit der Kategorie 3 zusammengefasst wurde. Dies lässt sich inhaltlich begründen: Wenn ein System in Verbindung mit einer Lernaktivität in mindestens der Hälfte der Unterrichtsstunden benutzt wird, kann davon ausgegangen werden, dass die Schüler*innen es als gewohnten Bestandteil des Mathematikunterrichts erleben. Die entstehende Kategorie bezeichnen wir als Kategorie 3 (durchgängige Nutzung). Die Nutzungshäufigkeit ist damit in drei Abstufungen pro Lernaktivität erfasst: $1=($ fast $)$ keine Nutzung ${ }^{1}, 2$ = gelegentliche Nutzung und 3 =durchgängige Nutzung. Die Angaben variieren zwar zwischen den Lerngelegenheiten und lassen auch auf unterschiedliche Nutzungsintensitäten der Lehrkräfte schließen, aber es zeigen sich empirisch keine unterschiedlichen Nutzungsprofile. Daher wurden die Angaben für die folgenden Analysen so aggregiert, dass die Nutzungsintensität abgebildet ist. Dazu wurde pro Lehrkraft die höchste angegebene Nutzungshäufigkeit ermittelt und die Lehrkraft damit der Gruppe der Nicht-Nutzung, der gelegentlichen Nutzung oder der durchgängigen Nutzung zugeordnet. Die aggregierte Nutzungshäufigkeit spiegelt also die höchste Angabe jeder Lehrkraft. Die Verteilungen der Nutzungshäufigkeit von DGS/CAS sind Abbildung 3 zu entnehmen.

Abb. 3: Nutzungshäufigkeiten von CAS und DGS nach Lernaktivitäten

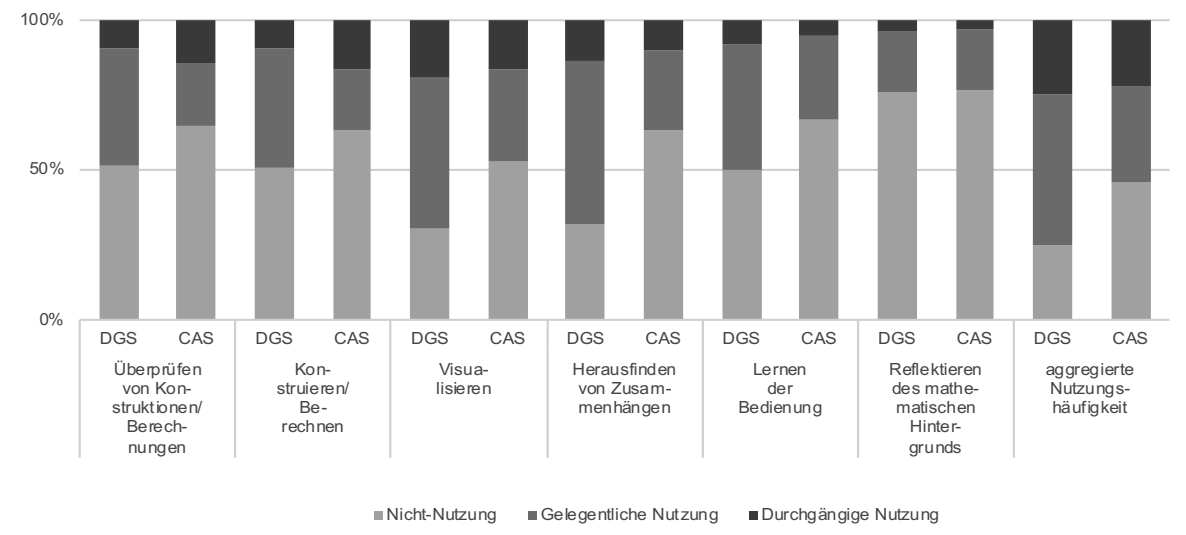

Quelle: eigene Darstellung

1 Im Folgenden wird zur besseren Lesbarkeit von der Nicht-Nutzung gesprochen. 
Nur wenige Lehrkräfte berichten von einer durchgängigen Nutzung, wobei sich der geringere Verpflichtungsgrad von CAS gegenüber DGS erwartungsgemäß in den aggregierten Nutzungshäufigkeiten niederschlägt.

\subsection{Welche Rolle spielen individuelle und organisationale Merkmale für die Nutzungshäufigkeit (Forschungsfrage 2)?}

Da DGS und CAS trotz unterschiedlichem Verbindlichkeitsgrad der gleichen Fachkultur unterliegen, ist zu vermuten, dass die Mechanismen, die zu Nutzungsunterschieden führen, dieselben sind. Deswegen wurde der Zusammenhang von Merkmalen der individuellen und der organisationalen Ebene mit der Nutzungshäufigkeit auf Basis der Daten zu beiden Systemen gemeinsam untersucht. Für den gestuften Vergleich der Gruppen "Nicht-Nutzung" mit "gelegentliche Nutzung" und "gelegentliche Nutzung" mit „durchgängige Nutzung" wurden zwei MLRs durchgeführt, die sich in den Referenzgruppen unterschieden (Referenz Nicht-Nutzung bzw. gelegentliche Nutzung). Es werden hier die Ergebnisse für benachbarte Gruppen berichtet.

In das finale Modell gingen die schulinternen Regelungen bezüglich DGS/CAS, der Zugang zu CAS/DGS sowie die Selbstwirksamkeitserwartung in Bezug auf das Unterrichten mit digitalen Medien als signifikante Prädiktoren ein $\left(\mathrm{R}_{\text {Cox\&snell }}^{2}=.20\right.$, $\mathrm{R}^{2}{ }_{\text {Nagelkerke }}=.22$, Modell $\left.\chi^{2}(6)=49.86, \mathrm{p}<.001\right)$. Nicht aufgenommen wurde die Einstellungs-Skala, da diese nicht zur Erklärung der Nutzungshäufigkeit beitrug.

\subsubsection{Gelegentliche vs. Nicht-Nutzung}

In dieser Studie erhöht das Vorhandensein schulinterner Regelungen bzgl. der genutzten Geräte die Wahrscheinlichkeit, dass Lehrkräfte Medien gelegentlich im Unterricht einsetzen (vgl. Tab. 4 auf der folgenden Seite). Das Odds Ratio von 4.06 deutet an, dass im Vergleich eine Lehrkraft, deren Schule interne Regelungen hat, mit einer vierfach höheren Chance zur Gruppe "gelegentliche Nutzung" statt zur Gruppe „Nicht-Nutzung“ zählt $(\mathrm{p}<.001)$. Die Selbstwirksamkeitserwartung und die Zugangsmerkmale zeigen in diesem Kontrast keinen Effekt. Das Vorhandensein schulinterner Regelungen scheint den Einstieg in die Nutzung der Systeme zu erleichtern.

\subsubsection{Durchgängige vs. Gelegentliche Nutzung}

Wird die durchgängige Nutzung mit der gelegentlichen Nutzung kontrastiert, steigt die Wahrscheinlichkeit, Medien durchgängig zu nutzen, um etwa das Vierfache, wenn 
die Zugangshürde zu den CAS/DGS niedrig ist (vgl. Tab. 4). Zudem zeigt sich ein Effekt der Selbstwirksamkeitserwartung in Bezug auf das Unterrichten mit digitalen Medien. Schulinterne Regelungen zeigen im Kontrast durchgängiger Nutzung mit gelegentlicher Nutzung keinen signifikanten Effekt. Förderlich für eine durchgängige Nutzung im Kontrast zur gelegentlichen Nutzung erscheinen ein einfacher Zugang zu den Systemen und die Einschätzung, mit digitalen Medien gut unterrichten zu können.

Tab. 4: Parameterschätzer der Gruppenvergleiche

\begin{tabular}{|c|c|c|c|c|c|c|}
\hline & \multicolumn{3}{|c|}{$\begin{array}{l}\text { Nicht-Nutzunga vs. } \\
\text { gelegentliche Nutzung }\end{array}$} & \multicolumn{3}{|c|}{$\begin{array}{l}\text { gelegentliche Nutzunga vs. } \\
\text { durchgängige Nutzung }\end{array}$} \\
\hline & $\stackrel{\mathrm{b}}{(S E)}$ & $\begin{array}{l}\text { Odds } \\
\text { Ratio }\end{array}$ & $p$-Wert & $\begin{array}{l}\mathrm{b} \\
(S E)\end{array}$ & $\begin{array}{l}\text { Odds } \\
\text { Ratio }\end{array}$ & $p$-Wert \\
\hline Konstanter Term & $\begin{array}{l}-.93 \\
(0.88)\end{array}$ & & .290 & $\begin{array}{l}-3.85 \\
(1.09)\end{array}$ & & $<.001$ \\
\hline $\begin{array}{l}\text { Vorhandensein } \\
\text { schulinterner Re- } \\
\text { gelungen }\end{array}$ & $\begin{array}{l}1.40 \\
(0.36)\end{array}$ & 4.06 & $<.001$ & $\begin{array}{l}-0.56 \\
(0.40)\end{array}$ & 0.57 & .153 \\
\hline Zugang & $\begin{array}{l}-0.26 \\
(0.41)\end{array}$ & 0.77 & .520 & $\begin{array}{l}1.38 \\
(0.38)\end{array}$ & 3.99 & $<.001$ \\
\hline $\begin{array}{l}\text { Selbstwirksam- } \\
\text { keitserwartung }\end{array}$ & $\begin{array}{l}0.26 \\
(0.32)\end{array}$ & 1.30 & .422 & $\begin{array}{l}1.07 \\
(0.36)\end{array}$ & 2.92 & .003 \\
\hline
\end{tabular}

Anm.: $\quad{ }^{a}$ Referenzkategorie

Quelle: eigene Berechnung

\section{Diskussion}

Wie stellt sich die Nutzung digitaler mathematikspezifischer Medien am Beispiel von CAS und DGS aktuell dar? Die Ergebnisse der Lehrkräftebefragung zeigen, dass vielfach noch nicht von einer durchgängigen Nutzung der Systeme auszugehen ist, wobei sich der unterschiedliche Verbindlichkeitsgrad von DGS und CAS in den Angaben der Lehrkräfte spiegelt. Zu beachten ist dabei, dass eine hohe Nutzungshäufigkeit noch keinen Schluss auf die unterrichtliche Qualität des Medieneinsatzes zulässt, da für die Beurteilung der Qualität der lernzielorientierte Einsatz zu berücksichtigen ist (Petko, 2012). Mit einer sporadischen Nutzung der durchaus komplexen Systeme können die in den Curricula intendierten Lernziele aber sicher nicht erreicht werden.

Die Ergebnisse zu den Bedingungsfaktoren deuten an, dass, ähnlich den Erkenntnissen aus fachunspezifischer Sicht, auch für die Nutzung mathematikspezifischer Medien organisationale Merkmale und Personenmerkmale zur Erklärung von Nutzungsunterschieden beitragen. Die Studie kann darüber hinaus Einsichten zur 
Relevanz verschiedener Faktoren im gestuften Vergleich Nicht-Nutzung - gelegentliche Nutzung - durchgängige Nutzung geben.

Auf individueller Ebene gehen in dieser Studie Einstellungen gegenüber den Medien als Prädiktor für die Mediennutzung nicht in das finale Modell ein, obwohl sie aus handlungstheoretischer Sicht relevant sind (zsf. Davis, 1989). Da für die Einstellungen in der vorliegenden Studie teils Deckeneffekte auftraten, differenzieren diese möglicherweise nicht ausreichend, um über die anderen Variablen hinaus Unterschiede in den Nutzungshäufigkeiten erklären zu können. Zu berücksichtigen ist dabei, dass die Einstellungen nicht medienspezifisch erhoben wurden. Es muss daher an dieser Stelle offenbleiben, ob Einstellungen gegenüber mathematikspezifischen Medien sich gegebenenfalls als zusätzliche Prädiktoren eignen.

Wie zu erwarten, trägt in dieser Studie die Selbstwirksamkeitserwartung der Lehrkräfte bezüglich des Medieneinsatzes zur Erklärung der Nutzungshäufigkeit bei. Jedoch wird die Selbstwirksamkeitserwartung erst im Vergleich der gelegentlichen mit der durchgängigen Nutzung relevant. Dasselbe gilt auch für Merkmale des Zugangs zu den mathematikspezifischen Medien, sodass niedrige organisationale Hürden erst für Unterschiede zwischen gelegentlicher und durchgängiger Nutzung relevant scheinen, nicht jedoch für die zwischen Nicht-Nutzung und gelegentlicher Nutzung. Einschränkend ist festzuhalten, dass weitere, in umfassenderen Studien als relevant identifizierte Merkmale auf organisationaler Ebene in dieser Studie nicht erhoben wurden (z. B. Qualität der Ausstattung, technischer Support).

Für die Erklärung von Unterschieden zwischen der Nicht-Nutzung und der gelegentlichen Nutzung erweisen sich in unserer Studie deutlich schulinterne fachspezifische Regelungen als wichtig. Es kann davon ausgegangen werden, dass das Vorhandensein solcher Regelungen impliziert, dass sich das Fachkollegium über die Geräte ausgetauscht hat, Vereinbarungen zur Nutzung in Klassenarbeiten ausgehandelt und die Geräte und deren Funktionen bestenfalls in das schulinterne Fachcurriculum eingegliedert sind. Deswegen verstehen wir die Frage nach den Regelungen bezüglich genutzter Geräte als einen Indikator für die an der Schule herrschende Kultur in Bezug auf CAS- bzw. DGS-Nutzung. Dies könnte seine Wirkung als Prädiktor im Einklang mit den Befunden zur Relevanz von schulorganisatorischen Merkmalen wie gemeinsamen Zielen und Visionen erklären.

Zu den Limitationen der Studie gehört, dass die Stichprobe eine Gelegenheitsstichprobe und somit nicht repräsentativ ist, auch wenn nicht von einer systematischen Verzerrung der Stichprobe im Vergleich zu einer repräsentativen Lehrkräftebefragung auszugehen ist. Weiter basiert die Studie auf Selbstberichten, sodass insbesondere keine objektivierbaren Angaben zu Nutzungshäufigkeiten vorliegen. Die Erhebung wurde zwar mit Bezug zu kanonischen Unterrichtsthemen durchgeführt, um die Validität relativer Vergleiche zu erhöhen, jedoch wissen wir nicht, wann die Lehrkräfte diese 
Themen zuletzt unterrichtet haben. Inwiefern die Generalisierbarkeit der Ergebnisse durch die nicht repräsentative Stichprobe und eingeschränkte Themenwahl beeinträchtigt ist, ist jedoch kaum abzuschätzen, da allgemein Daten zur Nutzung fachspezifischer Medien fehlen. Die Vermutung liegt aber nahe, dass die Nutzungsangaben die aktuelle Lage eher überschätzen, da angenommen werden kann, dass die Stichprobe insgesamt aus eher engagierten Lehrkräften besteht.

Für die Praxis lassen sich mehrere Ansatzpunkte zur Förderung von Mediennutzung im Mathematikunterricht begründen. Einerseits deuten die Ergebnisse an, dass Strategien zur Lehrkräftequalifizierung, die zu einer erhöhten Selbstwirksamkeitserwartung beitragen, sowie eine Senkung der Zugangshürden zu den Geräten förderlich sein können, um die Nutzungshäufigkeit von Medien im Fachunterricht zu steigern, wenn diese bereits genutzt werden. Andererseits legen die Ergebnisse nahe, dass Strategien, die auf Ebene der Schule und der Fachkollegien mit dem Ziel der Veränderung einer medienspezifischen schulinternen Fachkultur ansetzen, bei der Integration von Medien im Mathematikunterricht helfen können. In der Studie bildet sich das im relativ erklärmächtigen Indikator der schulinternen Regelungen in Bezug auf die genutzten Geräte für CAS und DGS ab. Inwiefern unspezifischere Maßnahmen, wie z.B. fachübergreifende Medienkonzepte, ebenfalls zur Erklärung der mathematikspezifischen Mediennutzung beitragen können, kann diese Studie nicht beantworten. Die Ergebnisse deuten aber an, dass eine Veränderung der fachspezifischen Medienkultur gerade für den Einstieg in die Nutzung mathematikspezifischer Medien hilfreich ist. Insgesamt implizieren die Ergebnisse der Studie, dass fachspezifische Maßnahmen, die auf der organisationalen Ebene ansetzen, mit solchen, die die individuelle Ebene adressieren, Hand in Hand gehen sollten.

Wir danken der Joachim Herz Stiftung für die Förderung des Projekts „MiU - Medieneinsatz im mathematisch-naturwissenschaftlichen Unterricht", in dessen Rahmen die Befragung stattfand, sowie allen Teilnehmenden der Befragung.

\section{Literatur und Internetquellen}

Ackeren, I. van, Aufenanger, S., Eickelmann, B., Friedrich, S., Kammerl, R., Knopf, J., et al. (2019). Digitalisierung in der Lehrerbildung. Herausforderungen, Entwicklungsfelder und Förderung von Gesamtkonzepten. DDS - Die Deutsche Schule, 111 (1), 103-119. https://doi.org/10.31244/dds.2019.01.10

Barzel, B. (2012). Computeralgebra im Mathematikunterricht: Ein Mehrwert - aber wann? Münster: Waxmann.

BITKOM (2015). Digitale Schule - vernetztes Lernen. Ergebnisse repräsentativer Schülerund Lehrerbefragungen zum Einsatz digitaler Medien im Schulunterricht. Zugriff am 22.04.2020. Verfügbar unter: https://www.bitkom.org/Bitkom/Publikationen/DigitaleSchule-und-vernetztes-Lernen.html.

Davis, F. D. (1989). Perceived Usefulness, Perceived Ease of Use, and User Acceptance of Information Technology. MIS Quarterly, 13, 319-340. https://doi.org/10.2307/249008 
Drossel, K., Eickelmann, B., \& Gerick, J. (2017). Predictors of Teachers' Use of ICT in School - the Relevance of School Characteristics, Teachers' Attitudes and Teacher Collaboration. Education and Information Technologies, 22 (2), 551-573. https://doi. org/10.1007/s10639-016-9476-y

Eickelmann, B., Bos, W., Gerick, J., Goldhammer, F., Schaumburg, H., Schwippert, K. et al. (2019). ICILS 2018 \#Deutschland. Computer- und informationsbezogene Kompetenzen von Schülerinnen und Schülern im zweiten internationalen Vergleich und Kompetenzen im Bereich Computational Thinking. Münster: Waxmann.

Eickelmann, B., Gerick, J., \& Koop, C. (2017). ICT Use in Mathematics Lessons and the Mathematics Achievement of Secondary School Students by International Comparison: Which Role do School Level Factors Play? Education and Information Technologies, 22 (4), 1527-1551. https://doi.org/10.1007/s10639-016-9498-5

Fahlgren, M., \& Brunström, M. (2014). A Model for Task Design with Focus on Exploration, Explanation, and Generalization in a Dynamic Geometry Environment. Technology, Knowledge and Learning, 19 (3), 287-315. https://doi.org/10.1007/s10758-014-9213-9

Handal, B., Cavanagh, M., Wood, L., \& Petocz, P. (2011). Factors Leading to the Adoption of a Learning Technology: The Case of Graphics Calculators. Australasian Journal of Educational Technology, 27 (2), 343-360. https://doi.org/10.14742/ajet.974

Härtig, H., Kampschulte, L., Lindmeier, A., Ostermann, A., Ropohl, M., \& Schwanewedel, J. (2018). Wie lässt sich Medieneinsatz im Fachunterricht beschreiben? In M. Ropohl, A. Lindmeier, H. Härtig, L. Kampschulte, A. Mühling \& J. Schwanewedel (Hrsg.), Medieneinsatz im mathematisch-naturwissenschaftlichen Unterricht (S. 157-192). Hamburg: Joachim Herz Stiftung.

Hermans, R., Tondeur, J., van Braak, J., \& Valcke, M. (2008). The Impact of Primary School Teachers' Educational Beliefs on the Classroom Use of Computers. Computers \& Education, 51 (4), 1499-1509. https://doi.org/10.1016/j.compedu.2008.02.001

Hillmayr, D., Reinhold, F., Ziernwald, L., \& Reiss, K. (2017). Digitale Medien im mathematisch-naturwissenschaftlichen Unterricht der Sekundarstufe: Einsatzmöglichkeiten, Umsetzung und Wirksamkeit. Münster: Waxmann.

Hoffkamp, A. (2011). The Use of Interactive Visualizations to Foster the Understanding of Concepts of Calculus: Design Principles and Empirical Results. ZDM, 43 (3), 359-372. https://doi.org/10.1007/s11858-011-0322-9

Huberman, M. (1991). Der berufliche Lebenszyklus von Lehrern. Ergebnisse einer empirischen Untersuchung. In E. Terhart (Hrsg.), Unterrichten als Beruf. Neuere amerikanische und englische Arbeiten zur Berufskultur und Berufsbiographie von Lehrerinnen und Lehrern (S. 249-267). Köln: Böhlau.

KMK (Sekretariat der Ständigen Konferenz der Kultusminister der Länder in der Bundesrepublik Deutschland). (2015). Bildungsstandards im Fach Mathematik für die Allgemeine Hochschulreife. Beschluss der KMK vom 18.10.2012. Berlin.

KMK (Sekretariat der Ständigen Konferenz der Kultusminister der Länder in der Bundesrepublik Deutschland). (2016). Strategie der Kultusministerkonferenz „Bildung in der digitalen Welt“. Beschluss der Kultusministerkonferenz vom 8.12.2016. Berlin.

Lindau, B., Kübler, S., \& Spada H. (2013). Entwicklung und Überprüfung eines Modells der Bereitschaft zum Medien- und Technologieeinsatz bei weiblichen und männlichen Lehramtsstudierenden. Unterrichtswissenschaft, 41, 20-37.

Lorenz, R., Bos, W., \& Endberg, M. (2017). Schule digital - der Länderindikator 2017. Schulische Medienbildung in der Sekundarstufe I mit besonderem Fokus auf MINTFächer im Bundesländervergleich und Trends von 2015 bis 2017. Münster: Waxmann.

Meinhardt, C., Rabe, T., \& Krey, O. (2016). Selbstwirksamkeitserwartungen in physikdidaktischen Handlungsfeldern. Skalendokumentation. Version 1.0 (Februar 2016). Zugriff am 10.04.2020. Verfügbar unter: http://nbn-resolving.de/urn:nbn:de:0111-pedocs-118180. 
Petko, D. (2012). Teachers' Pedagogical Beliefs and their Use of Digital Media in Classrooms: Sharpening the Focus of the 'Will, Skill, Tool' Model and Integrating Teachers' Constructivist Orientations. Computers \& Education, 58, 1352-1359. https://doi. org/10.1016/j.compedu.2011.12.013

Prasse, D. (2012). Bedingungen innovativen Handelns in Schulen. Funktion und Interaktion von Innovationsbereitschaft, Innovationsklima und Akteursnetzwerken am Beispiel der IKT-Integration an Schulen. Münster: Waxmann.

Rieß, M. (2018). Zum Einfluss digitaler Werkzeuge auf die Konstruktion mathematischen Wissens. Wiesbaden: Springer. https://doi.org/10.1007/978-3-658-20644-4

Rolfes, T., Roth, J., \& Schnotz, W. (2020). Learning the Concept of Function with Dynamic Visualizations. Frontiers in Psychology, 11, 693. https://doi.org/10.3389/fpsyg.2020. 00693

Sailer, M., Murböck, J., \& Fischer, F. (2017). Digitale Bildung an bayerischen Schulen Infrastruktur, Konzepte, Lehrerbildung und Unterricht. München: vbw.

Schmidt-Thieme, B., \& Weigand, H.-G. (2015). Medien. In R. Bruder, L. Hefendehl-Hebeker, B. Schmidt-Thieme \& H.-G. Weigand (Hrsg.), Handbuch der Mathematikdidaktik (S. 461-490). Berlin: Springer. https://doi.org/10.1007/978-3-642-35119-8_17

Schmitz, G. S., \& Schwarzer, R. (2000). Selbstwirksamkeitserwartung von Lehrern: Längsschnittbefunde mit einem neuen Instrument. Zeitschrift für Pädagogische Psychologie, 14 (1), 12-25. https://doi.org/10.1024//1010-0652.14.1.12

Thomas, M. O. J., \& Palmer, J. M. (2014). Teaching with Digital Technology: Obstacles and Opportunities. In A. Clark-Wilson, O. Robutti \& N. Sinclair (Hrsg.), The Mathematics Teacher in the Digital Era: An International Perspective on Technology Focused Professional Development (S. 71-89). Dordrecht: Springer Netherlands. https://doi. org/10.1007/978-94-007-4638-1_4

Thurm, D. (2020). Digitale Werkzeuge im Mathematikunterricht integrieren. Zur Rolle von Lehrerüberzeugungen und der Wirksamkeit von Fortbildungen. Wiesbaden: Springer. https://doi.org/10.1007/978-3-658-28695-8

Tondeur, J., van Keer, H., van Braak, J., \& Valcke, M. (2008). ICT Integration in the Classroom: Challenging the Potential of a School Policy. Computers \& Education, 51 (1), 212-223. https://doi.org/10.1016/j.compedu.2007.05.003

Venturini, M., \& Sinclair, N. (2017). Designing Assessment Tasks in a Dynamic Geometry Environment. In A. Leung \& A. Baccaglini-Frank (Hrsg.), Digital Technologies in Designing Mathematics Education Tasks (S.77-98). Cham: Springer International Publishing. https://doi.org/10.1007/978-3-319-43423-0_5

Weigand, H.-G., \& Bichler, E. (2010). Towards a Competence Model for the Use of Symbolic Calculators in Mathematics Lessons: The Case of Functions. ZDM, 42 (7), 697-713. https://doi.org/10.1007/s11858-010-0274-5

Anje Ostermann, geb. 1991, Doktorandin am Leibniz-Institut für die Pädagogik der Naturwissenschaften und Mathematik in Kiel.

E-Mail: ostermann@leibniz-ipn.de

Korrespondenzadresse: Olshausenstraße 62, 24118 Kiel

Hendrik Härtig, Prof. Dr., geb. 1979, Professor für Didaktik der Physik an der Universität Duisburg-Essen.

E-Mail: hendrik.haertig@uni-due.de

Korrespondenzadresse: Universitätsstr. 2, 45141 Essen 
Lorenz Kampschulte, Dr., geb. 1976, Leiter der Hauptabteilung Bildung am Deutschen Museum in München.

E-Mail: 1.kampschulte@deutsches-museum.de

Korrespondenzadresse: Museumsinsel 1, 80538 München

Mathias Ropohl, Prof. Dr., geb. 1981, Professor für Didaktik der Chemie an der Universität Duisburg-Essen.

E-Mail: mathias.ropohl@uni-due.de

Korrespondenzadresse: Schützenbahn 70, 45127 Essen

Julia Schwanewedel, Prof. Dr., geb. 1979, Professorin für Didaktik der Naturwissenschaften - Biologie an der Universität Hamburg.

E-Mail: julia.schwanewedel@uni-hamburg.de

Korrespondenzadresse: Von-Melle-Park 8, 20146 Hamburg

Anke Lindmeier, Prof. Dr., geb. 1979, Professorin für Didaktik der Mathematik an der Friedrich-Schiller-Universität Jena.

E-Mail: anke.lindmeier@uni-jena.de

Korrespondenzadresse: Ernst-Abbe-Platz 2, 07743 Jena 


\section{Anhang}

\section{Skala „Selbstwirksamkeitserwartung beim Unterrichten mit digitalen Werkzeugen“}

\section{Verwendete Skala}

\begin{tabular}{|c|c|c|c|c|c|}
\hline & $\begin{array}{l}\text { Bitte bewerten Sie, inwiefern Sie den folgenden Aussagen } \\
\text { zustimmen. }\end{array}$ & 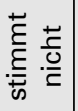 & 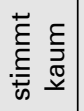 & 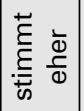 & 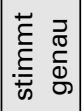 \\
\hline digM1 & $\begin{array}{l}\text { Ich kann in meiner Unterrichtsplanung zu den Lernzielen } \\
\text { passende Einsätze digitaler Medien planen, auch wenn mei- } \\
\text { ne Schule nicht optimal mit digitalen Medien ausgestattet } \\
\text { ist. }\end{array}$ & $\square$ & $\square$ & $\square$ & $\square$ \\
\hline digM2 & $\begin{array}{l}\text { Ich kann den Einsatz digitaler Medien im Fachunterricht so } \\
\text { planen, dass meine Schülerinnen und Schüler begeistert } \\
\text { sind, auch wenn sie sich sonst wenig für das Fach interes- } \\
\text { sieren. }\end{array}$ & $\square$ & $\square$ & $\square$ & $\square$ \\
\hline digM3 & $\begin{array}{l}\text { Ich kann den Einsatz eines digitalen Mediums im Fachunter- } \\
\text { richt didaktisch begründen, auch wenn ich dieses digitale } \\
\text { Medium noch nicht selbst eingesetzt habe. }\end{array}$ & $\square$ & $\square$ & $\square$ & $\square$ \\
\hline digM4 & $\begin{array}{l}\text { Ich kann eine Lösung für technische Probleme beim Unter- } \\
\text { richten mit digitalen Medien finden, auch wenn ich unter } \\
\text { Zeitdruck stehe. }\end{array}$ & $\square$ & $\square$ & $\square$ & $\square$ \\
\hline digM5 & $\begin{array}{l}\text { Ich kann den Einsatz digitaler Medien so gestalten, dass } \\
\text { meine Schülerinnen und Schüler motiviert sind mitzuar- } \\
\text { beiten, auch wenn es sich um eine unbeliebte Randstunde } \\
\text { handelt. }\end{array}$ & $\square$ & $\square$ & $\square$ & $\square$ \\
\hline digM6 & $\begin{array}{l}\text { Ich kann den fachlichen Lernprozess durch den Einsatz di- } \\
\text { gitaler Medien unterstützen, auch wenn unvorhergesehene } \\
\text { Verständnisschwierigkeiten auftreten. }\end{array}$ & $\square$ & $\square$ & $\square$ & $\square$ \\
\hline
\end{tabular}




\section{Konzeption der Skala}

\begin{tabular}{|l|l|l|}
\hline & \multicolumn{2}{|l|}{$\begin{array}{l}\text { Entsprechung nach Meinhardt, Rabe \& Krey (2016). Selbstwirksamkeitserwartungen } \\
\text { in physikdidaktischen Handlungsfeldern, Handlungsfeld Experimentieren (SWE-EX), } \\
\text { Dimensionen Planung (exp) und Durchführung (exd) }\end{array}$} \\
\hline digM1 & exp1 & $\begin{array}{l}\text { Ich kann in meiner Unterrichtsplanung zu den Lernzielen passende } \\
\text { Experimente aufbauen, auch wenn die Physiksammlung schlecht aus- } \\
\text { gestattet ist. }\end{array}$ \\
\hline digM2 & exp6 & $\begin{array}{l}\text { Ich kann ein Experiment planen, das meine Schülerinnen und Schüler } \\
\text { begeistert, auch wenn sie sich sonst wenig für Physik interessieren. }\end{array}$ \\
\hline digM3 & $\begin{array}{l}\text { Keine direkte Entsprechung, enthält den Aspekt der didaktischen Begründung ohne } \\
\text { hohe Vertrautheit wie in exp7 }\end{array}$ \\
\hline digM4 & exd5 & $\begin{array}{l}\text { Ich kann ein Experiment, das im Physikunterricht nicht auf Anhieb funk- } \\
\text { tioniert, zum Laufen bringen, auch wenn ich unter Zeitdruck stehe. }\end{array}$ \\
\hline digM5 & exd8 & $\begin{array}{l}\text { Ich kann ein Experiment so inszenieren, dass meine Schülerinnen und } \\
\text { Schüler motiviert sind mitzuarbeiten, auch wenn es sich um eine unbe- } \\
\text { liebte Randstunde handelt. }\end{array}$ \\
\hline digM6 & Keine direkte Entsprechung, enthält Aspekte von exd6, exd7 und exd9 \\
\hline
\end{tabular}

